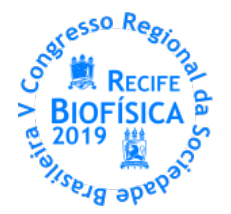

\title{
USO DE ARTÉRIAS UMBILICAL HUMANA COMO MÉTODO ALTERNATIVO PARA USO DE ANIMAIS EM ESTUDOS ELETROFISIOLÓGICOS: AVALIAÇÃO DO EFEITO MIORRELAXANTE DO CITRAL.
}

\author{
Débora de Menezes Dantas ${ }^{2 *}$, Luís Pereira de Morais ${ }^{2}$, Andressa de Alencar Silva ${ }^{2}$, Carla Mikevely de Sena Bastos ${ }^{1}$, \\ Maria Franciele Lima Gomes ${ }^{1}$, Renata Evaristo Rodrigues da Silva ${ }^{2}$, Thaís de Souza Amorim¹, Roseli Barbosa ${ }^{2}$ \\ ${ }^{1}$ Graduanda do curso de Ciências Biológicas da Universidade Regional do Cariri - URCA; \\ ${ }^{2}$ Laboratório de Fisiofarmacologia das Células Excitáveis - LFCE, URCA. \\ *debora_fdj@hotmail.com
}

\begin{abstract}
INTRODUÇÃO
A grande maioria dos estudos que envolvem a investigação da ação de substâncias de origem natural, terapeuticamente promissora e associadas às fisiopatologias que acometem a musculatura lisa, utiliza-se de modelos de músculo liso isolados de animais de laboratórios, onde dependem unicamente desse método para o desenvolvimento de pesquisas. Entretanto, o uso disseminado desses seres vivos na pesquisa tem sido alvo de diversas discussões, principalmente de caráter ético, em função do grande número de animais requerido e do sofrimento causado durante os experimentos (WHITE, 2001). Por esse motivo, a reavaliação desse método nos experimentos é uma tendência mundial e cada vez mais vem se procurando por novas alternativas que substituam o uso de animais em instituições de ensino, tanto para aulas práticas como no âmbito da pesquisa cientifica.
\end{abstract}

Essas ações tiveram início a partir do surgimento do programa 3Rs, assim denominado em função das iniciais, em inglês, de seus principais objetivos: redução (Reduction), refinamento (Refinement) e substituição (Replacement). Este programa foi iniciado pela federação das Universidades para o Bem-estar Animal (The Universities Federation for Animal Welfare - UFAW's), que de forma resumida significa reduzir o número de animais utilizados na pesquisa, refinar a metodologia de forma a diminuir a dor e desconforto sofrido por os mesmos, e buscar métodos alternativos que, por fim, substituam seu uso, sendo esta a meta máxima a ser alcançada (RUSSEL; BURCH 1992; CAZARIN, et al., 2004).

Neste contexto, visando substituir o uso de animais laboratoriais, obedecendo assim a regra dos 3 Rs (reduce, refine, replace), o Laboratório de Fisiofarmacologia das Células Excitáveis da Universidade Regional do Cariri - URCA, vem implementando métodos alternativos utilizando vasos de cordões umbilicais humanos que seriam descartados. Os experimentos estão sendo desenvolvidos com a técnica de banho de órgãos, verificando o efeito vasorrelaxante de produtos naturais como citral (3,7dimetil-2,6-octadienal). Este monoterpeno acíclico natural, é uma junção de dois monoterpenos aldeídos acíclicos isômeros geométricos - geranial e neral (SADDIQ, KHAYYAT, 2010), pode ser encontrado como componente majoritário do $\mathrm{OE}$ de diversas plantas aromáticas, empregadas na medicina popular como as espécies de Melissa officinalis (MENEZES et al., 2015), Lippia alba (STASHENKO et al., 2014), Zingiber officinale (FASS et al., 2014) Cymbopogon citratus (PASSOS et al., 2012). O citral apresenta várias atividades terapêuticas relatadas na literatura dentre elas 0 efeito vasorrelaxante em artéria aorta de ratos (DA SILVA et al., 2018).

Dessa forma, o estudo do citral em artéria umbilical humana tornase necessário para que possamos disponibilizar dados sobre sua ação no músculo liso vascular humano, podendo este revelar-se como um possível alvo terapêutico para o tratamento de transtornos HUA, como a pré-eclâmpsia. Vale ressaltar que o cordão umbilical é um material biológico humano prontamente disponível, descartado após o parto, fonte abundante de tecido vascular humano e que possui baixo custo. Além disso, seu uso facilita ainda as pesquisas clinicas, uma vez que, o tecido utilizado é humano.

\section{MATERIAIS E MÉTODOS}

Inicialmente o projeto foi aprovado pelo comitê de ética e pesquisa da URCA, com parecer: 1.962.667, onde visa substituir a utilização de artéria aorta de ratos por artéria umbilical de humanos, uma vez que este tecido é descartado pelo próprio hospital. Várias porções do cordão umbilical, de 10 a $12 \mathrm{~cm}$, foram obtidos de partos normais no bloco de obstetrícia do Hospital e Maternidade São Francisco de Assis que está localizado no município de Crato-CE, os tecidos somente são coletados com o consentimento prévio das doadoras. Os cordões foram colhidos e colocados em uma solução salina fisiológica, Tyrode modificado. No Laboratório de Fisiofarmacologia das Células Excitáveis, as artérias foram isoladas de seus tecidos anexos e da geleia de Worth, em seguida realizou-se a montagem dos anéis com 3 à 4 $\mathrm{mm}$ de comprimentos, em equipamento de banho de órgãos isolados (FIGURA 1). 0 tecido foi submetido a estabilização de $2 \mathrm{~h}$ sob tensão de aproximadamente $1,5 \mathrm{~g}$. Após a estabilização, foram evocadas contrações com os agentes contraturantes $\mathrm{KCl}(60 \mathrm{mM})$ e 5-HT $(10 \mu \mathrm{M})$, em experimentos distintos, com intervalos de lavagem entre eles. Posteriormente concentrações cumulativas do citral $(1-1000 \mu \mathrm{g} / \mathrm{mL})$ foram administradas sobre o platô de contração na presença de $\mathrm{KCl}$ e 5-HT.

\section{RESULTADOS E DISCUSSÃO}

Para avaliar a influência do citral sobre as contrações induzidas por $\mathrm{KCl}(60 \mathrm{mM})$, foi administrado concentrações crescentes e cumulativas do citral (1-1000 $\mu \mathrm{g} / \mathrm{mL})$. Nesse estudo, verificou-se que o citral foi capaz de relaxar $100 \%$ de forma dependente da concentração, as preparações de artérias pré-contraídas por $\mathrm{KCl}$ $(60 \mathrm{mM})$ quando comparado com o controle. Observou-se que o efeito do citral ocorreu de forma estatisticamente significante a 
partir da contração de $300 \mu \mathrm{g} / \mathrm{mL}(\mathrm{p}<0,007$, ANOVA, método Holm-Sidak). $O$ valor de $\mathrm{IC}_{50}$ obtido foi: $398,56 \pm 9,48 \mu \mathrm{g} / \mathrm{mL}$ (FIGURA 2).

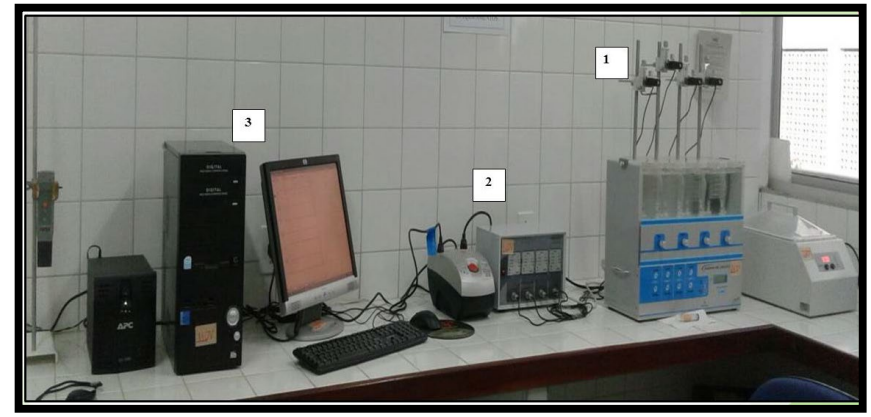

Figura 1. Imagem do equipamento utilizado para medir a resposta mecânica do músculo liso. 01: Transdutores de força mecânica acoplados a hastes fixas de sustentação; 02: Banho de órgãos de quatro cubetas com capacidade para $10 \mathrm{~mL}$; 03: Interface de 4 canais conversora de sinal mecânico em sinal analógico digital; 04:Modulo isolador estabilizado; e 05: Monitor responsável por abrigar software WINDAQ. (Fonte: DA SILVA, 2018).

Ao final foram feitas lavagens consecutivas durante 30 minutos para posteriormente contrair com $\mathrm{KCl}$ mostrando reversão e viabilidade do tecido depois do experimento.

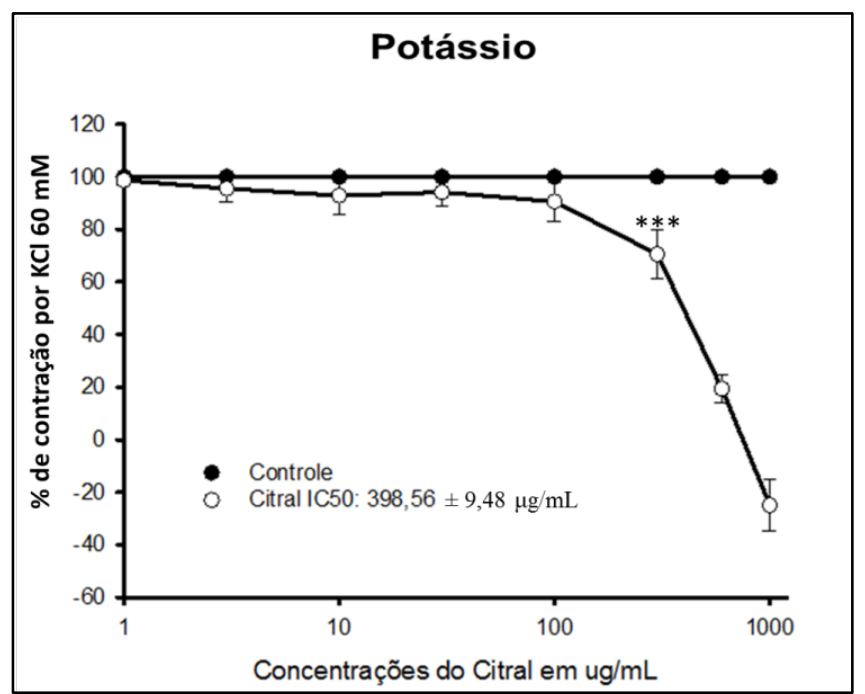

Figura 2. Gráfico concentração-resposta do efeito relaxante produzido pelo citral em preparação de tecido de artéria umbilical humana pré-contraida com $\mathrm{KCl}(60 \mathrm{mM})$. A concentração do citral utilizada foi de $1000 \mu \mathrm{g} / \mathrm{mL}$. Os valores estão expressos como média \pm E.P.M.; * representa os efeitos significantes $(p<0,05$, one-way ANOVA seguido de Holm-Sidak).

Nos estudos onde a contração da musculatura lisa de artéria umbilical foi evocada por 5-HT $(10 \mu \mathrm{M})$, foi administrado concentrações crescentes e cumulativas do citral $(1-1000 \mu \mathrm{g} / \mathrm{mL})$. Observou-se que o citral também promoveu um relaxamento que assim como na via do potássio foi estatisticamente significante a partir da contração de $300 \mu \mathrm{g} / \mathrm{mL}(\mathrm{p}<0,007$, ANOVA, método Holm-Sidak), com valor de $\mathrm{IC}_{50}: 843,1 \pm 6,80 \mu \mathrm{g} / \mathrm{mL}$, porém não obteve eficácia máxima (FIGURA 3).

Ao final foram feitas lavagens consecutivas durante 30 minutos para posteriormente contrair com $\mathrm{KCl}$ mostrando reversão e viabilidade do tecido depois do experimento.

Esse estudo demonstra que o citral bloqueia as contrações induzidas por potássio (via eletromecânica) e serotonina (via farmacomecânica). Onde observou-se que a atividade vasodilatadora do citral foi mais potente nas artérias contraídas com $\mathrm{KCl}$, apresentando eficácia máxima, revertendo totalmente a contração do músculo liso de artéria umbilical humana. Isso demonstra que o citral apresenta maior potencial no acoplamento eletromecânico.

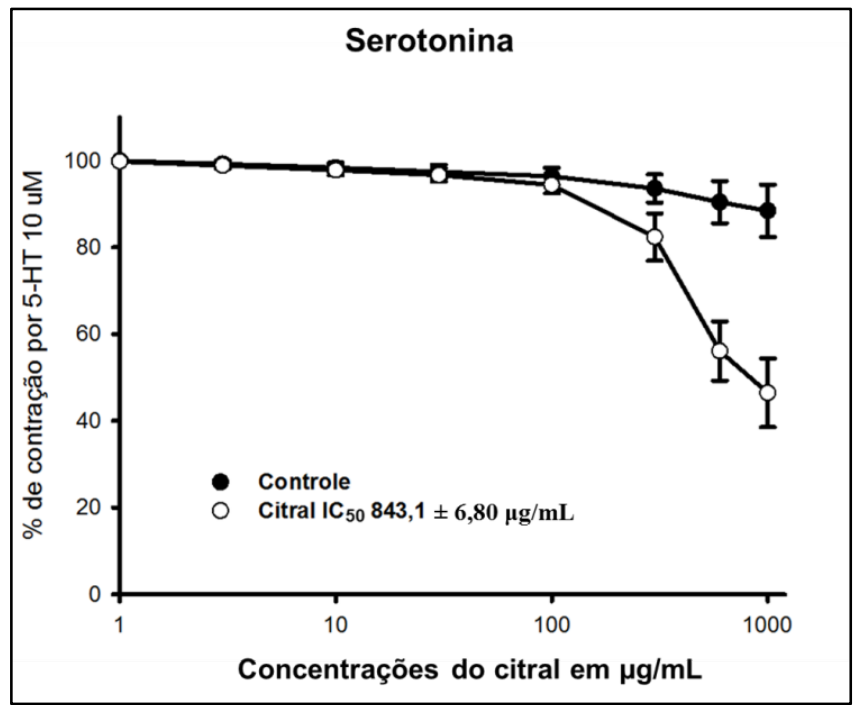

Figura 3. Gráfico concentração-resposta do efeito relaxante produzido pelo citral em preparação de tecido de artéria umbilical humana pré-contraida com 5-HT $(10 \mu \mathrm{M})$. A concentração do citral utilizada foi de $1000 \mu \mathrm{g} / \mathrm{mL}$. Os valores estão expressos como média \pm E.P.M.; * representa os efeitos significantes ( $p<0,05$, one-way ANOVA seguido de Holm-Sidak).

Da Silva et al., (2018), investigaram o efeito vasorelaxante do óleo essencial de Lippia alba (OELa) e seu constituinte majoritários citral em aorta isolada de ratos. Corroborando com os nossos resultados, nesse estudo o citral foi capaz de relaxar na via eletromecânica os anéis de aorta contraídos com $\mathrm{KCl}(60 \mathrm{mM})$, apresentando significância nas concentrações de 300, 600 e 1000 $\mu \mathrm{g} / \mathrm{mL}$. Pereira-de-Morais et al., (2019), demonstraram que o citral promoveu o relaxamento em útero isolado de ratas contraídos com $\mathrm{KCl}(60 \mathrm{mM})$, apresentando $\mathrm{IC}_{50}$ no valor $130,90 \pm 2,82 \mu \mathrm{g} / \mathrm{mL}$. Esses estudos fortalecem nossos achados em artéria umbilical humana, pois demonstram que o citral age na via do potássio.

Sadraei et al., (2003), verificaram que na via serotoninérgica, o óleo essencial de Melissa officinalis que possui o citral como constituinte principal, inibiram as contrações de íleo de rato induzidas por 5-HT. Nesse sentido, ainda na via seretoninergica, estudos realizados com traqueia isolada de ratos e íleo de cobaias, demonstram que o OELa que apresenta um elevado teor de citral, foi capaz de inibir contrações evocadas por 5-HT $(10 \mu \mathrm{M})$ (JARVIS et al., 2016). Esses estudos corroboram com os nossos resultados uma vez que demonstram que o citral age na vida da serotonina.

A maioria dos estudos para descoberta de novas substâncias com propriedade vasorelaxante ocorre em vasos de animais de laboratório (INGELFINGER, 2013). Em contrapartida, os resultados deste trabalho comprovam que os vasos umbilicais podem substituir o uso de animais em testes experimentais. Além do nosso trabalho também há estudos na literatura que utilizam esse modelo como Fernandes (2015) caracteriza os efeitos vasodilatador de nitratos orgânicos em artéria e veia isoladas de cordão umbilical humano, considerando os vasos umbilicais bons modelos para avaliação de substâncias vasoativas.

Os aspectos éticos da utilização de animais em experimentos estimulam a adoção de métodos alternativos sempre que possível e o uso desses seres vivos em situações de absoluta imprescindibilidade (BRASIL, 2008). Além da relevância em se adequar as questões éticas, a substituição por métodos alternativos ainda diminui os gastos gerados na criação e manutenção desses seres vivos em biotérios, o que vem a reduzir gastos para a universidade. 
Observamos o efeito relaxante mediado pelo citral, este foi reversível com a lavagem, demonstrando assim que esse monoterpeno não causa danos ao tecido humano. 0 uso de artéria umbilical humana apresenta-se como um excelente modelo para se avaliar substâncias vasoativas e com isso percebemos que é possível reduzir e substituir o número de animais eutanasiados com finalidade de pesquisa científica.

\section{CONCLUSÕES}

Dessa forma observou-se que o citral apresentou efeito miorrelaxante em artéria umbilical humana no acoplamento eletromecânico e farmacomecânico, sendo mais potente na via eletromecânica o que sugere estudos futuros para demonstrar seu mecanismo de ação. Vimos também que esse modelo vem confirmar uma alternativa para uma nova linha de pesquisa em substituição ao uso de animais na URCA, facilitando assim as pesquisas clinicas, uma vez que, o tecido utilizado é humano.

\section{REFERÊNCIAS}

CAZARIN, K. C. C.; CORRÊA, C. L.; ZAMBRONE, F. A. D. Redução, refinamento e substituição do uso de animais em estudos toxicológicos: uma abordagem atual. Revista Brasileira de Ciências Farmacêuticas, v. 40, n. 3, p. 289-299, 2004.

DA SILVA, R. E. R.; DE MORAIS, L. P.; SILVA, A. A.; BASTOS, C. M. S.; PEREIRA-GONÇALVES, Á.; KERNTOPF, M. R.; MENEZES, I. R. A.; LEAL-CARDOSO, J. H.; BARBOSA, R. Vasorelaxant effect of the Lippia alba essential oil and its major constituent, citral, on the contractility of isolated rat aorta. BIOMEDICINE \& PHAMACOTHERAPY, v. 108, p. 792-798, 2018.

FASS, L.; FELDER, M.; PATANKAR, M. S.; KAPUR, A. K. Citra lis the major component of ginger-derived terpenes to mediate p53dependent apoptosis in cancer cells. Cancer Research, v.74, p. 19, abstract $\mathrm{nr}$ 3211, 2014.

FERNANDES, M. C. A. Caracterização Do Efeito Vasodilatador Dos Nitratos Orgânicos GTN, NTHF, NCOE E BIS-NTHF Em Artéria E Veia Isoladas De Cordão Umbilical Humano. Porto Alegre: UFPB, 2015. Tese (Doutorado) - Programa de Pós- Graduação em Produtos Naturais e Sintéticos Bioativos, Universidade Federal da Paraíba, João Pessoa, 2015.

INGELFINGER, J. R. Experimental models of hypertension and their relevance to human hypertension. Pediatric Hypertension: Clinical hypertension and Vascular Diseases, p. 121-128, 2013.

JARVIS, G. E.; BARBOSA, R; THOMPSON, A. J. Noncompetitive Inhibition of 5-

HT3 Receptors by Citral, Linalool, and Eucalyptol Revealed by Nonlinear MixedEffects Modeling. Journal of Pharmacology and Experimental Therapeutics, v. 356, n. 3, p. 549-562, 2016.

MENEZES, C. P.; GUERRA, F. Q. S.; PINHEIRO, L. S.;TRAJANO, V. N.; PEREIRA, F. O.; LIMA, E. O. Investigation of Melissa officinalis L. Essential Oil for Antifungal Activity against Cladosporium carrionii. International Journal of Tropical Disease and Health, v. 8, n. 2, p. 49-56, 2015.

PASSOS, C. S.; CARVALHO, L. N.; PONTES JR, R. B.; CAMPOS, R. R.; IKUTA, O.; BOIM, M. Blood pressure reducing effects of Phalaris canariensis in normotensive and spontaneously hypertensive rats. Canadian journal of physiology and pharmacology, v. 90, n. 2, p. 201-208, 2012.

PEREIRA-DE-MORAIS, L; SILVA, A. A; DA SILVA, R. E. R; COSTA, S. R. H; MONTEIRO, Á. B; DOS SANTOS, C. R; DE SOUZA, A. T; DE MENEZES, I. R. A; KERNTOPF, M. R; BARBOSA, R. Tocolytic activity of the Lippia alba essential oil and its major constituents, citral and limonene, on the isolated uterus of rats. CHEMICO-BIOLOGICAL INTERACTIONS, v. 297, p. 155-155, 2018.
SADRAEI, H.; GHANNADI, A.; MALEKSHAHI, K. Relaxant effect of essential oil of

Melissa officinalis and citral on rat ileum contractions. Fitoterapia, v. 74 , n. 5 , p. 445

452, 2003.

STASHENKO, E. E.; MARTÍNEZ, J. R.; DURÁN, D.C.; CÓRDOBA, Y.; CABALLERO, D. Estudio comparativo de la composición química y la actividad antioxidante de los aceites esenciales de algunas plantas dél género Lippia (Verbenaceae) cultivadas em Colômbia. Revista de la Academia Colombiana de Ciências, v. 38, p. 89-105, 2014. WHITE, W. J. The use of laboratory animals in toxicologic research. On: HAYES, A. W. Principles and methods of toxicology, v. 4, p. 773-818, 2001. 